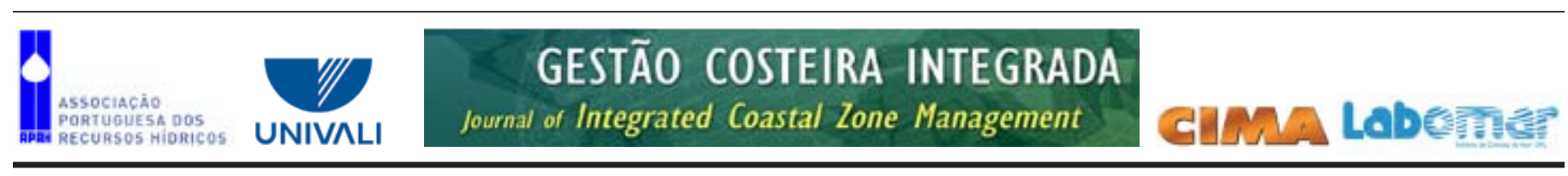

http://www.aprh.pt/rgci/pdf/rgci-345_Zacarias.pdf | DOI:10.5894/rgci345

\title{
Avaliação da capacidade de carga turística para gestão de praias em Moçambique: o caso da Praia do Tofo *
}

\author{
Tourism carrying capacity assessment for beach management in Mozambique: \\ the case of Praia do Tofo
}

\author{
Daniel Augusta Zacarias ${ }^{1}$
}

\section{RESUMO}

Os recursos costeiros constituem componente principal para o desenvolvimento de atividades recreativas. Entretanto, com o incremento destas atividades, as praias se vão transformando em espaços de congestáo humana que resulta na degradação dos ecossistemas locais e da capacidade de absorção dos impactos da comunidade circunvizinha, exigindo, deste modo, a introdução de práticas de gestáo mais apropriadas e direcionadas à gestão dos recursos. Considerando que a gestáo de visitantes em espaços costeiros deve ser rigorosamente planeada para se alcançarem os objetivos de conservação e gestão dos recursos e ao mesmo assegurar que os turistas alcançam uma experiência positiva e satisfazem as suas expectativas em relaçáo ao destino turístico, a determinação do número apropriado de pessoas que cada praia pode suportar é considerada uma tarefa primordial. Neste sentido, este artigo apresenta os resultados de avaliação da capacidade de carga turística da Praia do Tofo (aplicada para dois cenários) através da aplicação do Modelo de Avaliação da Capacidade de Carga idealizado por Cifuentes (1992), fundamentado em trabalho de campo, contagem de turistas e questionários, os resultados sugerindo que a praia do Tofo tem capacidade para acolher entre 896 e 447 turistas por dia sem deturpar as condiçóes naturais e ecológicas prevalecentes.

Palavras-chave: turismo, capacidade de carga, gestão de praias, Praia do Tofo

\section{ABSTRACT}

Coastal zones are important areas for recreational activities and are determinants of extensive tourist flows all over the World. Unfortunately these flows are a source of negative impacts in recreational settings resulting in beach overcrowding, beach erosion and degradation of coastal ecosystems. As such, beaches are being transformed into places of human congestion that results in degradation of local ecosystems and reduction of the capacity of the surrounding communities to absorb the impacts of tourism, thus requiring the introduction of more appropriate management practices directed to resources management. As coastal ecosystems have considerable impact on well-being, their sustainable management is also important. As such, ensuring human and ecosystem well-being in beaches is an important matter, especially considering that the amount of people living or using the coast is increasing year after year. With a heavy presence of humans in coastal recreational settings, the complexity of these habitats is affected and the management of visitors needs to be carefully planned in order to achieve the objectives of conservation and resource management and to ensure that tourists reach a positive experience and meet their expectations about the destination. Considering the sensitivity of coastal recreational settings, determining the appropriate number of people which can be supported at each individual beach is considered a primary task. This paper is an attempt to respond to this issue by presenting the results of a tourism carrying capacity study developed at Praia do

1 - Escola Superior de Hotelaria e Turismo de Inhambane. E-mail: Zacarias danieldream15@yahoo.com.br 
Tofo (southern Mozambique) by means of applying a combination of the Framework for Carrying Capacity Assessment as the guiding tool and several indicators for each category of carrying capacity (physical, real and effective carrying capacity). The field work was conducted at Praia do Tofo Beach in southern Mozambique from August $08^{\text {th }}$ to September 15 $5^{\text {th }} 2010$, involving measurement of the available area for leisure, tourists counts, direct interviews to tourists and evaluation of the infrastructural quality that support recreational activities. Based on two scenarios of beach utilization for tourists/ recreationists $\left(5 \mathrm{~m}^{2}\right.$ and $10 \mathrm{~m}^{2}$ per user), results indicate that the physical carrying capacity (considering as indicators the amount of land in $\mathrm{m}^{2}$ available for leisure, beach utilization and the rotation factor) limits the number of tourists/ recreationists to 57298 and 28649 visits/day (scenario 1 and 2 respectively) and the real carrying capacity (based on the real carrying capacity and correction factors - rainy and windy days, period of the year without sunshine and visual water quality) restrained beach utilization to 1,414 and 706 tourists/recreationists per day. Considering these parameters and the indicators of the management capacity (beach sanitation, parking facilities, beach accessibility, lifesaving facilities and the presence of domestic animals at the beach), the effective carrying capacity indicates that this beach can support a range of 896 and 447 tourists/recreationists a day (scenario 1 and 2 respectively) without distorting the prevailing natural and ecological conditions.

Keywords: tourism, carrying capacity, beach management, Tofo beach

\section{INTRODUÇÃO}

As áreas costeiras constituem ambientes preciosos para o estabelecimento de assentamentos humanos e atividades de desenvolvimento, principalmente o turismo. Tal como sugerido pelo Relatório Mundial de Recursos 2000-2001, as margens continentais são regióes de elevada produtividade biológica e boa acessibilidade, tendo sido centros de atividades humanas por milénios (UNDP et al., 2000). Como resultado, cerca de $40 \%$ da população mundial reside nos primeiros $100 \mathrm{~km}$ da linha costeira (apenas 22\% da terra firme planetária), e as projeçôes assumem um crescimento para cerca de 75\% em 2025 (Finkl \& Kruempfel, 2005). Este cenário, associado às projeçôes de crescimento do turismo, coloca as áreas costeiras em perigo de degradaçáo em ritmo cada vez maior, principalmente como resultado da associação dos impactos gerados pelas comunidades autóctones e dos turistas, resultando, por conseguinte, na deterioração das condiçôes atuais e completa alteração da capacidade de carga destas áreas.

Como referem Burke et al., (2000), globalmente cerca de três bilióes de pessoas vive na faixa costeira, aumentando em certas épocas do ano (especialmente no verão), com alguns países e regióes duplicando a sua populaçáo devido ao turismo. De forma ilustrativa, a costa mediterrânea acolhe cerca de 298 milhôes de turistas internacionais e $400 \mathrm{mil}$ turistas domésticos todos os anos (Weigert, 2012; Drakos \& Kutan, 2008, Perry, 2000); o estado da Flórida (nos Estados Unidos) recebe cerca de 85 milhóes de turistas (Houston, 2002, Dimanche \& Lepetic, 1999); o Brasil recebe 40 milhóes de turistas e na faixa costeira duplica a sua população no verão (Becker, 2001), as Caraíbas recebem cerca de 10 milhóes de turistas (Hinrichsen, 1999) e a cidade do Cabo recebe cerca de $49 \%$ dos fluxos turísticos que se dirigem para a África do Sul (Ballance et al., 2000). Com este potencial demonstrado pelas áreas costeiras, o principal desafio é a busca de soluções para integração dos fluxos turísticos e salvaguarda dos ecossistemas e valores culturais existentes nesta faixa, especialmente nos países com elevado potencial de atração turística, como é o caso de Moçambique.

Considerando que a beleza e a qualidade ambiental dos destinos turísticos são os principais fatores de atração dos segmentos costeiros (Manning, 1998), a implementação de estratégias apropriadas para gestão das atividades turísticas em praias constitui a base para gestão sustentável dos recursos e integração das aspirações comunitárias. Para o caso de Moçambique, a avaliação da capacidade de carga turística de praias se afigura primordial, especialmente considerando a visão e interesse de "fazer do turismo de praia e mar uma das fontes para a contribuiçáo na economia nacional numa base de práticas mundialmente aceites e ambientalmente sustentáveis" (MICOA, 2007:25). Considerando este pressuposto e a necessidade de gestão sustentável de ecossistemas comuns, este artigo pretendea presentar os resultados da avaliaçáo da capacidade de carga turística da praia do Tofo como contributo para gestáo sustentável do ambiente costeiro.

\section{BREVE DISCUSSÁO DO CONCEITO DE PRAIA E CAPACIDADE DE CARGA}

\subsection{A praia como destino turístico}

A abordagem científica define praias como "zonas de material náo consolidado que se estendem desde a linha de maré baixa até ao local onde se dá a alteração brusca dos materiais ou das formas fisiográficas ou até à linha de vegetação permanente" (Shore Protection Manual, 1984 apud Zacarias et al., 2011:1075). Estas áreas constituem importante fonte de receitas para muitos países devido ao crescimento absoluto do turismo que direciona as pessoas para a faixa costeira para usufruir da natureza, escapar à congestão urbana e para relaxar (Vaz et al., 2009, Williams $\&$ Micallef, 2009).

Como referem Silva et al., (2012), "em todo o mundo as praias com as suas diversas possibilidades de usos recreacionais, além do seu valor cénico e ecológico, constituem uma das principais motivaçôes para vultuosos investimentos". Integradas no conceito de turismo de sol e praia, as praias apresenta elevada sazonalidade de ocupação, "explicada fundamentalmente pelas características próprias do produto que se comercializa" (Sanchez \& Garcia, 2003), o que implica que o nível de utilização se concentra nos períodos que podem satisfazer as expetativas de desfrute das mesmas, como os meses mais quentes, com menos vento e sem chuva (Morgan et al., 1993, Jędrzejczak, 2004, Hamilton, 2007; Mansfeld et al., 2007).

Embora seja uma fonte de renda, a gestão do turismo de praia ainda não tem destaque na literatura económica (Hall, 
1997), tendo alguma expressão na literatura ecológica. Com o aumento dos fluxos de visitantes, a pressão sobre as condiçôes físicas, socioeconómicas e culturais também aumentam, exigindo, deste modo, a integraçáo das necessidades de todos os stakeholders no processo de planeamento sustentável, que segundo Papageorgiou \& Brotherton (1999) resulta na avaliação da capacidade de carga, um conceito fundamental para provisão de alternativas para gestáo da interação entre as atividades humanas e o meio ambiente.

\subsection{Avaliaçáo da capacidade de carga e gestáo costeira}

Em sequência ao anteriormente descrito, "o planeamento turístico deve ser direcionado de uma maneira ambientalmente sustentável, assegurando que asociedadegarantasuasobrevivência sem exceder a capacidade do ambiente, pois é deste que provêm os recursos e o contexto para a economia e o desenvolvimento social' (Oliveira, 2010:62). Neste sentido, a capacidade de carga assume um papel fundamental para gestão ambiental, especialmente em áreas sensíveis como é o caso de praias. Embora o debate atual imponha reservas em relação à validade deste conceito (McCool \& Lime, 2001; Mcleod, 1997), diversas são as opinióes de académicos e investigadores que defendem a necessidade de sua determinação (Butler, 1996, Brown et al., 1997; De Ruyck et al., 1997, Pereira da Silva, 2002).

Definida como "o número máximo de pessoas que pode visitar, em simultâneo, determinado destino turístico sem destruir as condiçóes físicas, ecológicas, económicas e socioculturais e sem causar redução inaceitável da satisfação dos visitantes" (OMT, 1981), a avaliação da capacidade de carga é utilizada para avaliar o impacto do turismo no espaço e no ambiente, sendo um mecanismo para estabelecimento de padrôes de sustentabilidade do turismo (Lim, 1998; Jovicic \& Dragin, 2008). A sua determinação requer informação relacionada com os recursos e a infraestrutura disponível, sendo extremamente influenciada pela sazonalidade, período do dia, qualidade dos recursos explorados, equipamentos existentes e a satisfação dos utilizadores (Clark, 1996; Ceballos-Lascurain, 1998).

Embora a capacidade de carga revele o número ótimo de visitantes que determinado destino pode acolher, esta deve ser considerada como o ponto em que a infraestrutura/ superestrutura e as condiçóes naturais se tornam insuficientes para satisfazer as necessidades da população residente e dos turistas, originando assim impactos negativos (Coccossis \& Parpairis, 1999; Batta, 2000; Coccossis, 2004; Kallis \& Coccossis, 2004).

$\mathrm{O}$ crescente interesse em relação à capacidade de carga é derivado do crescimento exponencial do turismo em combinação com o crescente aumento da consciência ambiental (MacLeod \&Cooper, 1992), sendo particularmente importante para a gestão de áreas costeiras que enfrentam mudanças bruscas como resultado da pressão demográfica (Kay \& Alder, 1999). Entretanto, a validade do conceito de capacidade de carga turística tem sido questionada, devido à elevada subjetividade associada à definição do número ótimo de utilizadores (MacLeod \& Cooper, 1992; Crofts \& Mather, 2001), um argumento que se enquadra no pressuposto de que a determinação da mudança aceitável constitui "um julgamento social baseado em aspetos científicos, mas desenvolvido considerando a envolvente política e ética (McCool \& Lime, 2001).

\section{ABORDAGEM METODOLÓGICA}

\subsection{Caracterizaçáo da área de estudo}

A Praia do Tofo (Figura 1) constitui um segmento costeiro enquadrado na faixa costeira a oeste da cidade de Inhambane e integrada na área prioritária para investimentos turísticos (APIT) que se estende do distrito de Zavala ao distrito de Massinga (MITUR, 2003). É uma praia com características rurais, natural e dominada por ondas com uma amplitude média de maré inferior a 3 metros (Hoguane, 2007), protegida por maciços recifes de coral existentes a alguns metros onshore. Esta praia foi descoberta durante o regime colonial durante os anos 60 , tendo as primeiras habitaçóes de veraneio sido implantadas na década de 70 . A tendência de ocupação do espaço na Praia do Tofo foi interrompida entre 1986 e 1992 devido à instabilidade política, tendo o processo reiniciado em 1994 através do investimento estrangeiro (maioritariamente sul-africano) que implantou hotéis, lodges, bares e escolas de mergulho na regiáo.

A Praia do Tofo é uma praia semi-encaixada (Oliveira, 2006), uma vez que se encontra abrigada por um promontório apresentando uma forma assimétrica, sendo constituída por uma zona de sombra próxima do promontório, protegida da ação direta das ondas e fortemente curvada, e a outra extremidade relativamente retilínea. Segundo Francisco (2008) este espaço caracteriza-se por ser uma praia oceânica maioritariamente arenoa e com dunas, apresentando algumas rochas, vegetação pioneira (rasteira), vegetação antropogénica e arbórea e habitaçóes de material convencional e de alvenaria (Figura 1).

\subsection{Metodologia de avaliaçáo da capacidade de carga}

A capacidade de carga turística foi determinada com base na adaptação e aplicaçấo do modelo de avaliação da capacidade de carga em áreas protegidas desenvolvido por Cifuentes (1992). Este modelo busca estabelecer o limite aceitável de visitas que determinado destino turístico pode suportar com base nas condiçóes físicas, biológicas e ecológicas da área, considerando três níveis principais: a capacidade de carga física, a capacidade de carga real e a capacidade de carga efetiva (Cifuentes et al., 1996; 1999). Para a sua aplicaçâo é importante considerar os fluxos turísticos, o tamanho do destino, o espaço ótimo disponível para cada turista se movimentar livremente e o intervalo de tempo gasto em média por cada turista no destino. Considerando as variáveis acima apresentadas, a capacidade de carga da área de estudo foi determinada seguindo três etapas principais (Figura 2): Com base neste modelo, a capacidade de carga física (CCf) é definida como o número máximo de visitantes que se podem acomodar em determinada área, em determinado tempo específico, sendo calculada com base na aplicaçáo da Equação 1:

$$
C C f=\frac{A}{A_{u}} \times F_{r}
$$




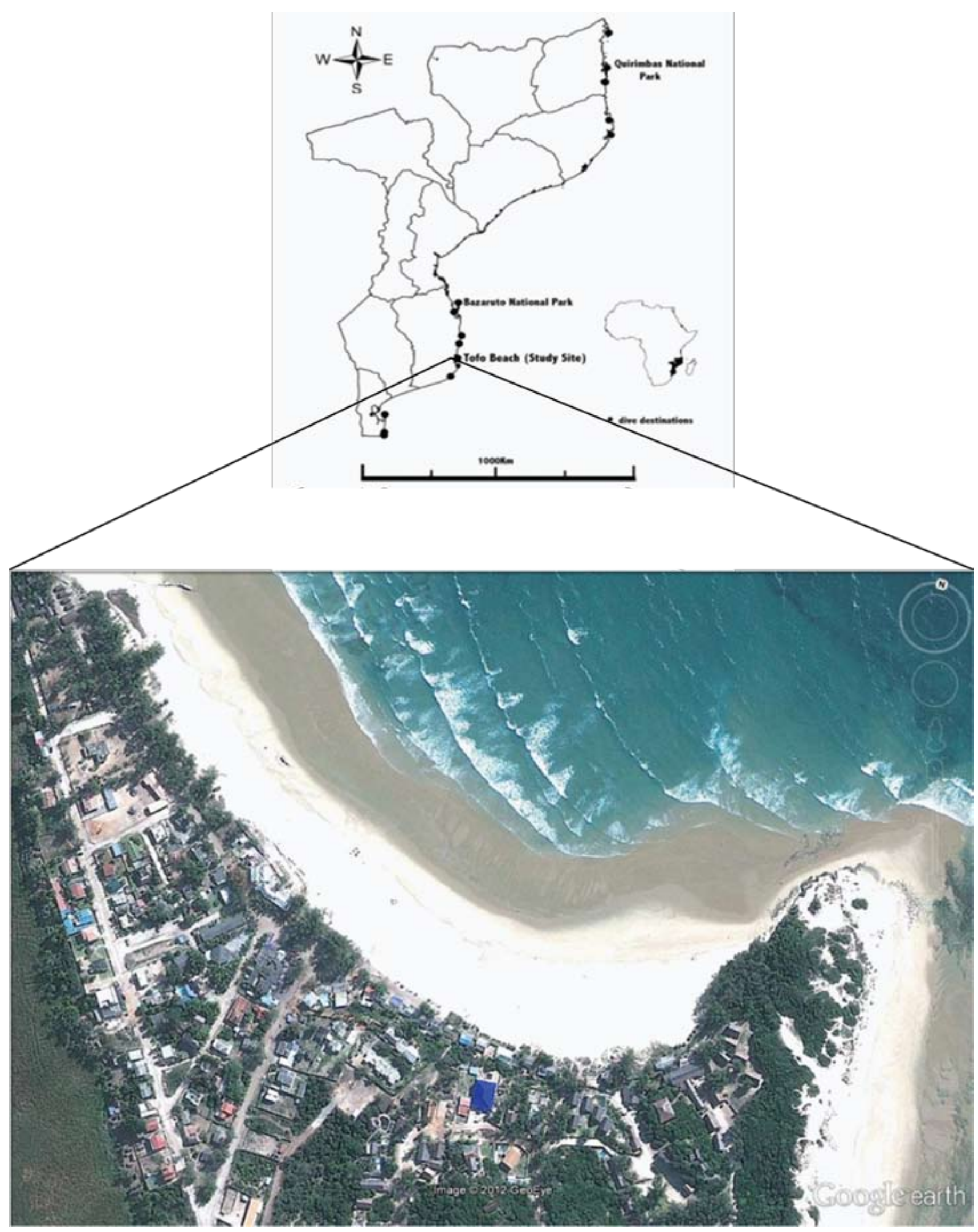

Figura 1. Localização espacial da área de estudo (imagem Google Earth)

Figure 1. Geographic location of the study area (Google Earth image) 


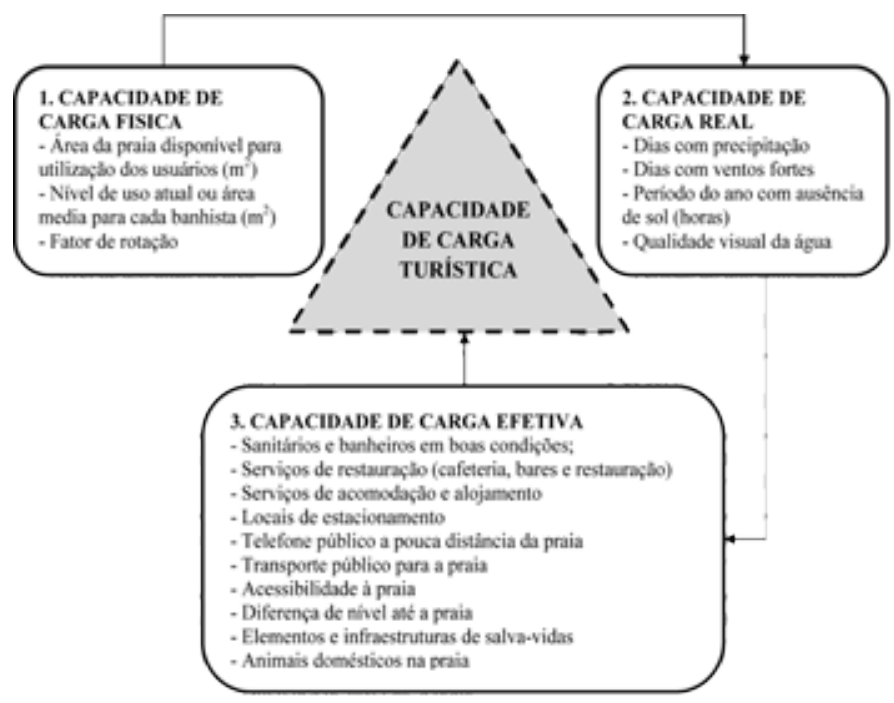

Figura 2. Etapas e variáveis de capacidade de carga adotadas neste estudo (Adaptado de Cifuentes, 1992)

Figure 2. Steps and carrying capacity variables adopted in this study (Adapted from Cifuentes, 1992)

Onde:

$\mathrm{CCf}=$ capacidade de carga física

$\mathrm{A}=$ tamanho da área de estudo

$\mathrm{A}_{\mathrm{u}}=$ área disponível para cada utilizador

$\mathrm{F}_{\mathrm{r}}$ = fator de rotação ou período médio de tempo de permanência de cada visitante na praia. Dados os constrangimentos impostos pela brevidade de execução do trabalho de campo, este valor não foi determinado, tendo sido adaptado de Silva et al. (2008) e De Ruyck et al. (1997), cujos estudos foram desenvolvidos em ambientes similares.

Uma vez determinada a CCF, foi necessário determinar a capacidade de carga real (CCr), ou seja o número máximo permissível de pessoas num determinado espaço após a incorporação de factores de correção (variáveis físicas, ambientais, ecológicas, sociais e de maneio) derivados das características intrínsecas do destino. Este procedimento deriva do facto a visita a determinada praia ser restringida por factores como a qualidade de água, insolação, acessibilidade, velocidade do vento, precipitação, dentre outros. Assim, a capacidade de carga foi calculada com base na Equação 2:

$C C r=C C f \times(f c 1 \times f c 2 \times f c 3 \times \ldots f c n)$

Onde:

$\mathrm{CCr}=$ capacidade de carga real

$\mathrm{CCf}=$ capacidade de carga física

$\mathrm{fc}=$ fatores de correção ou fatores restritivos da capacidade de carga ou visita a determinado espaço, determinados pela Equação 3:

$$
F c_{x}=\frac{M l_{x}}{M t_{x}}
$$

Onde:

$\mathrm{Fc}_{\mathrm{x}}$ = fator de correção da variável $\mathrm{x}$

$\mathrm{Ml}_{\mathrm{x}}=$ magnitude limitante da variável $\mathrm{x}$

$\mathrm{Mt}_{\mathrm{x}}=$ magnitude total da variável $\mathrm{x}$

Foram considerados fatores de correçáo da capacidade de carga neste estudo a precipitação, dias com ventos fortes, insolação (ausência) e qualidade visual da água. Após a determinação da CCr, calculou-se a capacidade de carga efectiva, ou seja, o número máximo de visitantes que o destino pode suportar considerando a CCR e a capacidade de gestão $(\mathrm{Cg})$, ou seja, "a média das condiçóes que a administração da área necessita ou dispóe para poder cumprir na íntegra as suas funçôes e objectivos" (Amador et al., 1996:19). Para calcular a CCe foi aplicada a Equaçáo 4:

$$
C C e=C C r \times C g
$$

Onde:

$\mathrm{CCe}=$ capacidade de carga efectiva

$\mathrm{CCr}=$ capacidade de carga real

$\mathrm{Cg}$ = capacidade de gestão, determinada a partir da avaliação da infraestrutura de apoio à gestáo de praias (acesso, estacionamento, contentores de lixo, chuveiros e banheiros). Neste estudo, o processo de avaliação da capacidade de gestáo fundamentou-se na análise dos indicadores apresentados no Tabela 1.

\section{RESULTADOS E DISCUSSÃO}

Os resultados das mediçóes de campo indicam que a Praia do Tofo ocupa, em maré baixa, uma área aproximada de $94496 \mathrm{~m}^{2}$, tendo fluxos turísticos concentrados na época quente (Setembro a Fevereiro) e na semana de Páscoa, embora seja possível encontrar turistas ao longo de todo o ano em resultado das temperaturas amenas do canal de Moçambique (temperaturas médias da água iguais ou superiores a $25^{\circ} \mathrm{C}$ ). Os resultados de contagem de turistas indicaram uma tendência de ocupação dos espaços localizados na retaguarda e no núcleo central da praia, facto que pode ser motivado pela existência de árvores de sombra que protegem os veraneantes do sol e pela prática de algumas atividades típicas destes ambientes como banhos de sol, passeio na areia e desportos de areia.

Com base na dimensão da área de estudo $\left(94496 \mathrm{~m}^{2}\right)$ e num factor de rotação igual a 3 (de Ruyck et al., 1997; Silva et al. 2008), a capacidade de carga física da Praia do Tofo (determinada para dois cenários de utilização: $5 \mathrm{~m}^{2} \mathrm{e}$ $10 \mathrm{~m}^{2}$ por utilizador) resultou numa variação entre 57298 pessoas/ dia e 28649 pessoas/ dia. Embora exorbitantes, estes números são demasiado teóricos e indicam, apenas, o número cumulativo de pessoas que pode ocupar a praia num dia considerando a divisão da mesma em polígonos de 
Tabela 1. Indicadores de qualidade de gestão aplicados na avaliação da capacidade de carga efetiva na Praia do Tofo (adaptado) Table 1. Indicators of the management capacity applied to assess the effective carrying capacity at Praia do Tofo (adapted)

\begin{tabular}{l|c|c|c}
\hline \multirow{2}{*}{ Indicadores } & \multicolumn{2}{c}{ Grau de atratividade } \\
\cline { 2 - 4 } & Baixo (1) & Médio (2) & Alto (3) \\
\hline Sanitários e banheiros em boas condiçóes & Ausentes & Poucos & Adequados \\
\hline $\begin{array}{l}\text { Serviços de restauração (cafeteria, bares e } \\
\text { restauração) }\end{array}$ & Ausentes & Poucos & Adequados \\
\hline Serviços de acomodaçáo e alojamento & Ausentes & Poucos & Adequados \\
\hline Locais de estacionamento & Ausentes & Poucos & Adequados \\
\hline Telefone público a pouca distância da praia & Ausentes & Poucos & Adequados \\
\hline Transporte público para a praia & Ausente & Restrito & Adequado \\
\hline Acessibilidade à praia & Inadequado & $\begin{array}{c}\text { Adequado (não } \\
\text { pavimentado }\end{array}$ & $\begin{array}{c}\text { Adequado } \\
\text { (pavimentado) }\end{array}$ \\
\hline $\begin{array}{l}\text { Diferença de nível até a praia } \\
\text { Elementos e infraestruturas de salva-vidas }\end{array}$ & $\begin{array}{c}\text { Desnível com escadas ou } \\
\text { rampa inadequada }\end{array}$ & $\begin{array}{c}\text { Desnível com } \\
\text { escada ou rampa } \\
\text { adequadas }\end{array}$ & \begin{tabular}{c} 
Sem desnível \\
\hline Animais domésticos na praia
\end{tabular} \\
\hline
\end{tabular}

Fonte: Adaptado e melhorado de Silva et al. (2012)

Source: Adapted and improved from Silva et al. (2012)

$5 \mathrm{~m}^{2}$ e $10 \mathrm{~m}^{2}$ para os cenários 1 e 2 , respectivamente. Como referem Aranguren et al. (2008), os valores da capacidade de carga física não podem ser tomados como indicadores para a planificação e gestão de praias, uma vez que a simples existência de espaço não constitui fator fundamental para o desenvolvimento do turismo, devendo ser equacionados outros aspectos que condicionam ou limitam o exercício das actividades de veraneio como os parâmetros ecológicos e naturais. Deste modo, a incorporação dos fatores de correção se afigura importante uma vez que expressam as várias condicionantes ao desenvolvimento das atividades dos veraneantes, ou seja, são factores que revelam as condiçôes para a prática da actividade turística.
Dados retirados de Reddy (1986) e Westerink (1996), indicam que Moçambique possui cerca de 120 dias de precipitação ao ano, 90 dias de ventos moderados a fortes e 4380 horas de insolação adequada para turismo, enquanto observaçóes visuais indicam que a área de estudo enfrenta uma semana por ano em que a qualidade de água é reduzida devido ao fenómeno do upwelling. Com estes dados, os fatores de correção foram determinados segundo a Tabela 2 :

Com base nestes indicadores, a capacidade de carga real da área de estudo resultou em 1414 pessoas/ dia (cenário 1) e 706 pessoas/ dia (cenário 2), o que revela que embora uma certa quantidade de turistas possa ser fisicamente contida na praia, o seu grau de interação com fenómenos ou atributos

Tabela 2. Fatores de correção da capacidade de carga real e sua importância na Praia do Tofo.

Table 2. ???

\begin{tabular}{lccc}
\hline Fatores de restrição & $\begin{array}{c}\text { Magnitude limitante } \\
(\mathbf{M l x})\end{array}$ & Magnitude total (Mtx) & $\begin{array}{c}\text { Fator de correçáo } \\
\mathbf{C f}=\mathbf{1}-(\mathbf{M l x} / \mathbf{M t x})\end{array}$ \\
\hline Precipitação & 120 dias/ ano & 365 dias & 0.6712 \\
\hline Ventos fortes & 90 dias/ ano & 365 dias & 0.75 \\
\hline Qualidade de água & 7 dias/ ano & 365 dias & 0.098 \\
\hline Ausência de insolação & 4380 horas/ ano & 8760 horas & 0.5 \\
\hline
\end{tabular}

Fonte: Elaborada pelo autor com base na aplicação da Equação 3 
naturais determina a redução da qualidade do destino, ou seja, à medida que o número de turistas aumenta, aumenta também a sensação de congestão, a capacidade de recuperação da praia reduz e a qualidade ambiental gradualmente decresce (Aranguren et al., 2008).

Considerando que a Praia do Tofo se enquadra numa área prioritária para investimentos turísticos e é uma praia aberta para turismo de massas, é de se esperar que esteja provida de equipamentos que apoiem o desenvolvimento das atividades turísticas e possam aumentar o grau de satisfação dos visitantes. Entretanto, a avaliação dos indicadores apresentados na Tabela 1, acima, revela uma situação diferente da esperada, na medida em que alguns indicadores não estão disponíveis ou não são adequados ao desenvolvimento sadio de atividades turísticas.

Os resultados da Tabela 3 revelam que a capacidade de gestão na Praia do Tofo é de 63.3\%. Integrada esta capacidade à capacidade de carga real, a capacidade de carga efetiva da Praia do Tofo (após aplicação da Equação 4) resultou num número máximo permissível de 896 e 447 turistas por dia (cenário 1 e 2, respetivamente), como ilustra a Tabela 4.

Anteriormente foi definido que determinar o número permissível de visitantes em determinado destino turístico não constitui o ponto final para a sua gestão. Obviamente, os impactos negativos advindos de determinada atividade não dependem em grande medida do número de pessoas que a realizam, mas da forma como estas atividades são realizadas.
Neste sentido o impacto causado por 447 pessoas pode em determinadas circunstâncias ser superior ao impacto causado por 896 outros utilizadores. Para o caso de praias, a simples caminhada em dunas de apenas 20 pessoas pode ser mais devastador do que o efeito causado por cerca de 10 vezes mais banhistas.

Esta característica fez com que durante muito tempo a capacidade de carga fosse um conceito estagnado e desacreditado, principalmente pela descrença das suas reais capacidades e pelas dificuldades de assimilação do "número mágico" como estratégia de gestão dos impactos causados pela visitação de determinado destino. Para reverter este cenário, vários outros mecanismos de gestâo foram desenvolvidos, como forma de avaliar a dimensão do impacto causado pelos números propostos pela capacidade de carga, tais como a gestão do impacto dos visitantes, os limites de mudança aceitável, a experiência dos visitantes e proteção dos recursos, dentro outros.

\section{CONCLUSÃO}

Neste artigo se apresenta uma primeira tentativa para determinação da capacidade de carga da Praia do Tofo e consequente garantir a sustentabilidade deste importante destino turístico costeiro. A aplicação do intervalo entre 447 e 896 turistas (intervalo ótimo de turistas que pode visitar a praia diariamente, baseado em cenários de utilização de $10 \mathrm{~m}^{2} /$ utilizador e $5 \mathrm{~m}^{2} /$ utilizador) deve se acautelada na medida em

Tabela 3: Descrição dos valores atribuídos aos indicadores de capacidade de gestão na Praia do Tofo e respetivos graus de atratividade Table 3: Description of the values attributed to each indicator of the management capacity at Praia do Tofo and associated levels of attractiveness

\begin{tabular}{|c|c|c|c|c|c|}
\hline Indicador & $\begin{array}{l}\text { Valor } \\
\text { atribuído }\end{array}$ & $\begin{array}{l}\text { Valor } \\
\text { ótimo }\end{array}$ & $\begin{array}{l}\text { Percentagem } \\
\text { válida }\end{array}$ & Descrição & $\begin{array}{c}\text { Grau de } \\
\text { atratividade }\end{array}$ \\
\hline $\begin{array}{l}\text { Sanitários e banheiros em boas } \\
\text { condiçôes }\end{array}$ & 1 & 3 & 33.3 & Ausentes & Baixo \\
\hline $\begin{array}{l}\text { Serviços de restauração } \\
\text { (cafeteria, bares e restauraçâo) }\end{array}$ & 3 & 3 & 100.0 & Quantidade adequada & Alta \\
\hline $\begin{array}{l}\text { Serviços de acomodação e } \\
\text { alojamento }\end{array}$ & 3 & 3 & 100.0 & Quantidade adequada & Alta \\
\hline Locais de estacionamento & 1 & 3 & 33.3 & Ausentes & Baixo \\
\hline $\begin{array}{l}\text { Telefone público a pouca } \\
\text { distância da praia }\end{array}$ & 2 & 3 & 66.7 & Poucos & Médio \\
\hline Transporte público para a praia & 3 & 3 & 100.0 & Disponibilidade adequada & Alto \\
\hline Acessibilidade à praia & 2 & 3 & 66.7 & Adequado (não pavimentado) & Médio \\
\hline Diferença de nível até a praia & 2 & 3 & 66.7 & $\begin{array}{l}\text { Desnível com escada ou } \\
\text { rampa adequada }\end{array}$ & Médio \\
\hline $\begin{array}{l}\text { Elementos e infraestruturas de } \\
\text { salva-vidas }\end{array}$ & 1 & 3 & 33.3 & Ausentes & Baixo \\
\hline Animais domésticos na praia & 1 & 3 & 33.3 & Frequentes & Baixo \\
\hline$\sum \mathrm{e} \boldsymbol{\mu}^{*}$ & 19 & 30 & 63.3 & & \\
\hline
\end{tabular}

* O somatório $(\boldsymbol{\Sigma})$ é aplicado às variáveis “valor atribuído" e “valor ótimo”, enquanto a média ( $\boldsymbol{\mu})$ é aplicada à percentagem válida 
Tabela 4. Quadro resumo da capacidade de carga turística da Praia do Tofo

Table 4. A summary of the tourist carrying capacity at Praia do Tofo

\begin{tabular}{lcc}
\hline Dimensão da capacidade & Cenário $\mathbf{1}\left(\mathbf{5} \mathbf{m}^{2} /\right.$ utilizador $)$ & Cenário $\mathbf{2}\left(\mathbf{1 0} \mathbf{m}^{2} / \mathbf{u t i l i z a d o r}\right)$ \\
\hline Capacidade de carga física & 57298 Visitas/ dia & 28649 Visitas/ dia \\
\hline Capacidade de carga real & 1414 Utilizadores/ dia & 706 Utilizadores/ dia \\
\hline Capacidade de carga efetiva & 896 Utilizadores/ dia & 447 Utilizadores/ dia \\
\hline
\end{tabular}

que dimensão do impacto gerado por este número de turistas ainda não foi cabalmente determinada e a simples imposição deste intervalo pode acarretar danos irreversíveis. Deste modo, recomenda-se a adoçáo de outros mecanismos de gestão do impacto, que associados ao intervalo determinado neste artigo permitirão o estabelecimento de intervençôes estratégicas mais eficientes para gestão da Praia do Tofo.

\section{AGRADECIMENTOS}

Este artigo é parte da dissertaçáo de mestrado do autor, apresentada à Universidade do Algarve, Faculdade de Ciências e Tecnologia. O autor agradece ao Programa Erasmus Mundus pela concessão da bolsa de estudos para frequência do programa de formação em Gestão de Água e Zonas Costeiras (Erasmus Mundus Water and Coastal Management). $\mathrm{O}$ autor agradece, sobremaneira, as sugestōes e opinióes transmitidas por dois revisores anónimos, que ajudaram a melhorar a qualidade do artigo.

\section{REFERÊNCIAS BIBLIOGRÁFICAS}

Amador, E.; Cayot, L.; Cifuentes, M.; Cruz, E.; Cruz, F. (1996) - Determinación de la capacidad de carga turística en los sitios de visita del Parque Nacional de Galápagos, Equador. 34p., Instituto Equatoriano Florestal e de Áreas Naturaise VidaSelvagem, Parque Nacional ReservaMariña de Galápagos, Puerto Ayora, Islas Galápagos, Ecuador. Disponível em http://www.unida.org.ar/Bibliografia/ documentos/Desarrollo_Sustentable/GST/modulo5/ Capac\%20Carga\%20Turist\%20Gal\%C3\%A1 pagos.pdf

Arangunen, J.; Moncada, J.A.; Naveda, J.; Rivas, D.; Lugo, C. (2008) - Evaluación de la capacidad de carga turística en la playa Conomita, Municipio Guanta, Estado Anzoátegui. Revista de Investigación (ISSN: 1010-2914) 32(64): 3161, Caracas, Venezuela. Disponível em http://www2. scielo.org.ve/scielo.php? script $=$ sci_pdf\&pid $=$ S1010-291 $42008000200003 \& \operatorname{lng}=\mathrm{es} \& n r m=\mathrm{iso} \&$ tlng=es

Ballance, A.; Ryan, P.G.; Turpie, J.K. (2000) - How much is a clean beach worth? The impact of litter on beach users in the Cape Peninsula, South Africa. South African Journal of Science (ISSN: 0038-2353) 96(5): 210 - 213.

Batta, R.N. (2000) - Tourism and the environment: a quest for sustainability with special reference to developing countries, and policy analysis on Himachal Pradesh. 248p., Indus Publishing, New Delhi, India. ISBN 81-7387-110-8
Becker, B.K. (2001) - Políticas e planejamento do turismo no Brazil. Caderno Virtual de Turismo (ISSN: 1677-6976), 1(1): 1-7, Rio de Janeiro, Brasil. Disponível em http:// www.ivt.coppe.ufrj.br/caderno/index.php?journal=cader no\&page $=$ article\&op $=$ view $\&$ path $\% 5 B \% 5 D=2 \&$ path $\%$ $5 \mathrm{~B} \% 5 \mathrm{D}=1$

Brown, K.; Turner, R.K.; Hameed, H.; Bateman,I.A.N.(1997)Environmental carrying capacity and tourism development in the Maldives and Nepal. Environmental Conservation, 24(4): 316-325. doi: 10.1017/S0376892997000428

Burke, L.; Kura, Y.; Kassem, K.; Revenga, C.; Spalding, M.; McAllister, D. (2001) - Pilot analysis of global ecosystems: coastal ecosystems. 77p., World Resources Institute, Washington, D.C., U.S.A. ISBN: 1-56973-458-5. Disponível em http://pdf.wri.org/page_coastal.pdf

Butler, R.W. (1996) - The concept of carrying capacity for tourist destinations: dead or merely buried? Progress in Tourism and Hospitality Research, 2(3-4): 283-293. doi: 10.1002/pth.6070020309

Ceballos-Lascuráin, H. (1996) - Tourism, ecotourism, and protected areas: The state of nature-based tourism around the world and guidelines for its development. 301 p., IV World Congress on National Parks and Protected Areas; Cambridge, UK. ISBN 2-8317-0124-4.

Cifuentes, M.A. (1992) - Determinación de capacidad de carga turística en áreas protegidas. 28p., Centro Agronómico Tropical de Investigación y Enseñanza, Turrialba, Costa Rica. ISBN: 9977-57-129-5. Disponível em http:// www.ulpgc.es/hege/almacen/download/23/23388/ articulocifuentes.pdf

Clark, J.R. (1996) - Coastal zone management handbook. 720p., CRC Press / Leweis Publishers, Boca Raton, Flórida, USA. ISBN: 978-1566700924.

Coccossis, H. (2004) - Sustainable tourism and tourism carrying capacity: a new context. In: H. Coccossis e A. Mexa (eds.), The Challenges of Tourism Carrying Capacity Assessment: Theory and Practice, pp. 3-14; Ashgate Publishing, Surrey, U.K. ISBN: 0-7546-3569-4.

Coccossis, H. and Parpairis, A. (1999) - Tourism and the environment: some observations on the concept of carrying capacity, pp. 91-106. In: H. Briassoulis; J. van der Straaten (eds.), Tourism and the Environment: Regional, Economic, Cultural, and Policy Issues. Kluwer Academic Publishers, Dordrecht, The Netherlands. ISBN 0-79236136-9. 
Crofts, R., Mather, A. (2001) - Beaches of Wester Ross. 145p., Countryside Commission for Scotland, Department of Geography, University of Aberdeen, Edinburgh, Scottish. Disponível em http://www.snh.org.uk/pdfs/publications/ commissioned_reports/beachesWesterRoss.pdf

De Ruyck, M.C.; Soares, A.G.; McLachlan, A. (1997) Social carrying capacity as a management tool for sandy beaches. Journal of Coastal Research, 13(3): 822-830. http://www.jstor.org/pss/4298675

Drakos, K.; Kutan, A.M. (2003) - Regional effects of terrorism on tourism in three Mediterranean countries. Journal of Conflict Resolution, 47(5): 621-641. doi: 10.1177/0022002703258198

Finkl, C.W.; Kruempel, C. (2005) - Threats, obstacles and barriers to coastal environmental conservation: societal perceptions and managerial positionalities that defeat sustainable development. In: Gomes, F.V.; Pinto, F.T; Neves, L.; Sena, A.; Ferreira, O. (eds.), Proceedings of the First International Conference on Coastal Conservation and Management in the Atlantic and Mediterranean, pp. 1112. Algarve, Portugal. ISBN: 972-8558-09-0

Francisco, F.G.A. (2008) - Estudo da erosáo costeira na praia do Tofo. 68p., Trabalho de Licenciatura. Universidade Eduardo Mondlane, Maputo, Moçambique. Não publicado

Hall, D. (1997) - The tourism debate and environmental scientists. Environmental Scientist (ISSN: 0966 8411), 6(5): $1-2$.

Hamilton, J.M. (2007) - Climate and the destination choice of German tourists. In: B. Amelung; K. Blazejczyk; A. Matzarakis (eds); Climate change and tourism assessment and copying strategies, pp.55-68, Maastrich, The Netherlands. ISBN: 978-00-023716-4. Disponível em http://www.urbanclimate.net/matzarakis/papers/Book_ Nato.pdf

Hinrichsen, D. (1998) - Coastal waters of the World: trends, threats and strategies. 298p., Island Press, Washington DC, USA. ISBN: 1-55963-383-2

Hoguane, A.M. (2007) - Perfil diagnóstico da zona costeira de Moçambique. Revista de Gestão Costeira Integrada (ISSN: 1646-8872), 7(1): 69-82, Lisboa, Portugal. Disponível em http://www.aprh.pt/rgci/pdf/rgci7_8_Hoguane.pdf

Houston, J. R. (2002) - The economic value of beaches: a 2002 update. Shore and Beach (ISSN: 0037- 4237), 70(1)9-12, American Shore \& Beach Preservation Association, San Francisco, CA, U.S.A. Disponível em http://www.wcu.edu/WebFiles/PDFs/Economic_Value_ of_Beaches_2002.pdf

Jędrzejczak, M.F. (2004) - The modern tourist's perception of the beach: is the sandy beach a place of conflict between tourism and biodiversity? In: G. Schernewski; N. Löser (eds.), Managing the Baltic Sea, pp.109-119; Coastline Reports 2; ISSN 0928-2734. Disponível em http://www. vliz.be/imisdocs/publications/64145.pdf

Jovicic, D.; Dragin, A. (2008) - The assessment of carrying capacity: a crucial tool for managing tourism effects in tourist destinations. TURIZAM (ISSN: 1821-1127), 12: 4-11, Novi Sad, Serbia. Disponível em http://www.dgt. uns.ac.rs/turizam/arhiva/vol1_1.pdf
Kallis, G.; Coccossis, H. (2004) - Theoretical reflections on limits, efficiency and sustainability: implications for tourism carrying capacity. In: H. Coccossis e A. Mexa (eds.), The Challenges of Tourism Carrying Capacity Assessment: Theory and Practice, pp.15-36; Ashgate Publishing, Surrey, U.K. ISBN: 0-7546-3569-4.

Kanji, F. (2006) - A global perspective on the challenges of coastal tourism. 18p., Coastal Development Centre, Bangkok, Thailand. Disponível em http://cdc.fish.ku.ac. th/workonweb/Global_coastal_tourism.pdf

Lim, L.C. (1998) - Carrying capacity assessment of Pulau Payar Marine Park, Malaysia. WWF Bay of Bengal Programme for Fisheries Management, Relatório BOBP/REP/79, 139p., Madras, India. Disponível em http://www.fao. org/docrep/X5626E/X5626E00.htm

Lindberg, K.; McCool, S.F. (1998) - A critique of environmental carrying capacity as a means of managing the effects of tourism development. Environmental Conservation, 25(4): 291-292. doi:10.1017/ S0376892998000368

Manning, R. (1998) - "To provide for the enjoyment": recreation management in the National Parks. George Wright Forum, 15: 6-20. Disponível em http://www.uvm. edu/parkstudieslaboratory/publications/2_Provide_4_ Enjoyment.pdf

Mansfeld, Y.; Freundlich, A.; Kutiel, H. (2007) - The relationship between weather conditions and tourists' perception of comfort: the case of the winter sun resort of Eilat. In: B. Amelung; K. Blazejczyk; A. Matzarakis (eds). Climate change and tourism assessment and copying strategies, pp.116-138, Maastrich, The Netherlands. ISBN: 978-00-023716-4. Disponível em http://www. urbanclimate.net/matzarakis/papers/Book_Nato.pdf

McCool, S.F.; Lime, D.W. (2001) - Tourism carrying capacity: tempting fantasy or useful reality? Journal of Sustainable Tourism, 9(5): 372-388. doi:10.1080/09669580108667409

McLeod, S.R. (1997) - Is the concept of carrying capacity useful in variable environments? Oikos, (ISSN 00301299) 79: 529-542. Disponível em http://www.jstor.org/ discover/10.2307/3546897?uid=3738680\&uid=2\&uid $=4 \& \operatorname{sid}=21102451335747$

MICOA (2007) - Estratégia ambiental para o desenvolvimento sustentável de Moçambique. Aprovada na IXa sessão do Conselho de Ministros. 65p., Ministério para a Coordenação da Ação Ambiental (MICOA), Maputo, Moçambique. Disponível em http://www.legisambiente.gov.mz/index. php?option=com_docman\&task=doc_view\&gid $=137$

Morgan, R.; Jones, T.C.; Williams, A.T. (1993) - Opinions and perceptions of England and Wales Heritage Coast beach users: some management implications from the Glamorgan Heritage Coast. Journal of Coastal Research, (ISSN 0749-0258) SI 36:1083-1093. Disponível em http://www.jstor.org/discover/10.2307/4298165?uid=3 738680 \&uid $=2 \&$ uid $=4 \&$ sid $=21102451335747$

Oliveira, F.S.B.F. (2006) - Modelação empírica da forma plana de praias: dois casos de estudo. 11p., $8^{\circ}$ Congresso da Água, Associação Portuguesa dos Recursos Hídricos, Lisboa, Portugal. Disponível em http://www.aprh.pt/ congressoagua2006/COMUNICACOES/106.PDF 
Oliveira, F.V. (2010) - Capacidade de carga em cidades históricas. Revista Brasileira de Pesquisa em Turismo, (ISSN: 1982-6125) 4(1):61-75, São Paulo, SP, Brasil. Disponível em http://www.revistas.univerciencia.org/ turismo/index.php/rbtur/article/viewFile/319/310

Papageorgiou, K.; Brotherton, I. (1999) - A management planning framework based on ecological, perceptual and economic carrying capacity: the case study of Vikos-Aoos National Park, Greece. Journal of Environmental Management, 56: 271-284. doi: 10.1006/ jema.1999.0285

Pereira da Silva, C. (2002) - Beach carrying capacity assessment: how important is it? Journal of Coastal Research, (ISSN 0749-0258) SI 36: 190-197. Disponível em http://www.science.ulst.ac.uk/ics2002/carlos $\% 20$ da\%20silva.pdf

Perry, A. (2000) - Impacts of climate change on tourism in the Mediterranean: adaptive responses. 11 p., Fondazioni Eni Enrico Mattei, Swansea, Wales. Disponível em http:// www.feem.it/userfiles/attach/Publication/NDL2000/ NDL2000-035.pdf

Reddy, S.J. (1984) - General climate of Mozambique. 43p., Instituto Nacional de Investigação Agronómica, Série Terra e Água, Maputo, Moçambique. Disponível em http://library.wur.nl/isric/fulltext/ISRIC_7169.pdf

Sanchez, A.G.; Garcia, F.J.A. (2003) - El turismo cultural y el de sol y playa: sustitutivos o complementarios? Cuadernos de Turismo (ISSN: 1139-7861) 11: 97-102, Universidad de Murcia, Murcia, Spain. Disponível em http://revistas. um.es/turismo/article/view/19581/18921

Saveriades, A. (2000) - Establishing the social carrying capacity for tourist resorts of the east coast of the Republic of Cyprus. Tourism Management, 21(2): 147-156. doi: 10.1016/S0261-5177(99)00044-8

Silva, I.R.; Bittencourt, A.C.S.P.; Dias, J.A.; Souza Filho, J.R. (2012) - Qualidade recreacional e capacidade de carga das praias do litoral norte do estado da Bahia, Brasil.
Revista de Gestão Costeira Integrada, 12(2): 131-146. doi: $10.5894 / \mathrm{rgci} 297$

Silva, J.S.; Leal, M.W.V.; Araújo, M.C.B.; Barbosa, S.C.T.; Costa, M.F. (2008) - Spatial and temporal patterns of use of Boa Viagem Beach, northeast Brazil. Journal of Coastal Research, 24(1A): 79-86. doi: 10.2112/05-0527.1

UNDP; UNEP; World Bank; WRI (2000) - A guide to World Resources 2000-2001: people and ecosystems, the fraying web of life. 276p., Elsevier Science, Washington DC, USA. ISBN: 0-08-0437818

Vaz, B.; Williams, A.T.; Silva, C.P.; Phillips, M. (2009) - The importance of user's perception for beach management. Journal of Coastal Research (ISSN 0749-0258) SI 56: 1164-1168

Weigert, M. (2012) - The challenges of tourism in the Mediterranean Region. 5p., Institut de prospective économique du monde méditerranéen. Paris, França. Disponível em http:/www.ipemed.coop/adminIpemed/ media/fich_article/1352394536_M.\%20WEIGERT_ The $\% 20$ Challenges $\% 20$ of\%20Tourism $\% 20$ in $\% 20$ the\%20Mediterranean\%20Region.pdf

Westerink, R.M. (1986) - Evaluation of monthly precipitation data of Mozambique. 24p., Instituto Nacional de Investigação Agronómica, Série Terra e Água, Maputo, Moçambique. Disponível em http://library.wur.nl/isric/ fulltext/ISRIC_22741.pdf

Williams, A.T.; Micallef, A. (2009) - Beach management: principles and practice. 480p., EarthScan, London, UK. ISBN: 978-1-84407-435-8

Zacarias, D.A.; Williams, A.T.; Newton, A. (2011) Recreation carrying capacity estimations to support beach management at Praia de Faro, Portugal. Journal of Applied Geography, 31: 1075-1081. doi: 10.1016/j. apgeog.2011.01.020 